\title{
Risk-Preference in Coin-Toss Games ${ }^{1}$
}

\author{
Clyde H. Coombs and David Edward Meyer ${ }^{2}$ \\ The University of Michigan, Ann Arbor, Michigan 48104
}

\begin{abstract}
It is hypothesized that an individual has a preferred unidimensional risk level in a coin-tossing game, and that his preferences are single-peaked over the risk scale. Risk was varied by increasing both the monetary denomination $(D=1 c$ to $\$ 1)$ and number of tosses $(N=1$ to 20$)$ involved in a game. The rank order preference data of 30 subjects within sets of games having either constant $D$ or $N$, single stimulus preference data, and pair comparison preferences between games supported these hypotheses. Data also supported the existence of a function $R_{0}[(D, N)]$ which maps games onto the risk scale and is monotone increasing in both arguments. However, the exact form of the function may vary, depending on the particular set of games from which the subject chooses.
\end{abstract}

The prevailing expectation theories of individual decision making in a risky situation (Edwards, 1954, 1955) all avoid the subjective variable of riskiness. However, this variable has been given some attention as a stimulus dimension relevant to choice (Coombs and Pruitt, 1960; Royden, Suppes, and Walsh, 1959). In these experimental studies, the variance or some other parameter of the frequency distribution of a game's possible outcomes was explored as a potential correlate of risk. The evidence supports the hypothesis that variance is a strong correlate of risk in simple game situations, but in more complex situations, the concept of risk is elusive and idiosyncratic (Wilcox, 1967). Thus we have chosen to treat the concept of risk as being undefined in a strict sense, although we do assume the existence of a variable which, in addition to expected value, mediates preferences in a consistent manner, can be experimentally manipulated in various ways, and for intuitive reasons might be identified as risk.

The present study considers a number of coin-tossing games which vary in two aspects: the coin denomination $(D)$ involved in a single toss, and the number of tosses $(N)$ composing the complete game. As an example, the game $(25 c, 5)$ represents a game in which 5 quarters are tossed all at once. For each coin landing heads, the game's owner is paid a quarter by a bank. For each tail, the bank is paid a quarter by the owner.

${ }^{1}$ This study was supported by USPHS Grant MH-04236 and NSF GB-6782.

${ }^{2}$ The authors wish to thank David Krantz, J. E. Keith Smith, and Amos Tversky for criticism of an early draft of this paper. 
We propose the following axioms about risk and risk-preferences among games $(D, N)$, for which expected value is zero.

A1. For games with expected value zero, there exists a real-valued risk function $R\left[\left(D_{1}, N_{1}\right),\left(D_{2} N_{2}\right)\right]$ which maps pairs of games onto a risk scale $r$, is symmetrical in its arguments, and is such that

(i) If $D_{1}<D_{1}{ }^{\prime}$ or $N_{1}<N_{1}^{\prime}$, then

$$
\begin{aligned}
& R\left[\left(D_{1}, N_{1}\right),\left(D_{2}, N_{2}\right)\right] \leqslant R\left[\left(D_{1}^{\prime}, N_{1}\right),\left(D_{2}, N_{2}\right)\right] \text { and } \\
& R\left[\left(D_{1}, N_{1}\right),\left(D_{2}, N_{2}\right)\right] \leqslant R\left[\left(D_{1}, N_{1}^{\prime}\right),\left(D_{2}, N_{2}\right)\right]
\end{aligned}
$$

(ii) If $0<\delta, 0<n \leqslant N_{1}$, and $D_{1} \cdot N_{1}=\left(D_{1}+\delta\right)\left(N_{1}-n\right)$ then

$$
R\left[\left(D_{1}, N_{1}\right),\left(D_{2}, N_{2}\right)\right] \leqslant R\left[\left(D_{1}+\delta, N_{1}-n\right),\left(D_{2}, N_{2}\right)\right]
$$

(iii) If $n \min \left(N_{1}, N_{2}\right)$ and $D_{1}<D_{2}$ then

$$
R\left[\left(D_{1}, N_{1}\right),\left(D_{2}, N_{2}\right)\right] \leqslant R\left[\left(D_{1}, N-n\right),\left(D_{2}, N_{2}+n\right)\right] .
$$

A2. For games with expected value zero, the risk of a single game $(D, N)$ is determined by the function $R_{0}[(D, N)] \equiv R[(D, N),(0,0)]$.

A3. For every individual there exists a unique ideal level of risk I in $r$, such that the pair of games $\left\{\left(D_{1}, N_{1}\right),\left(D_{2}, N_{2}\right)\right\}$ is strictly preferred to the pair of games

$$
\left\{\left(D_{1}^{\prime}, N_{1}^{\prime}\right),\left(D_{2}^{\prime}, N_{2}^{\prime}\right)\right\}
$$

if and only if $\left|R\left[\left(D_{1}, N_{1}\right),\left(D_{2}, N_{2}\right)\right]-I\right|<\left|R\left[\left(D_{1}{ }^{\prime}, N_{1}{ }^{\prime}\right),\left(D_{2}{ }^{\prime}, N_{2}{ }^{\prime}\right)\right]-I\right|$.

In general terms, Axioms $\mathrm{A} 1$ and $\mathrm{A} 2$ state that the risk of playing a game $(D, N)$, or pair of such games, increases with both the denominations and number of tosses involved. They also assert that $D$ is a stronger risk determinant than $N$, in the sense of Axiom A1 (ii) and Axiom A1 (iii).

The Axiom A3 assumes the existence of a single-peaked preference function (Luce and Raiffa, 1957) defined over the risk scale $r$, with $I$ being the most preferred risk level.

These axioms together yield a number of predictions about rank order, pair comparison, and single stimulus preferences within certain sets of games.

\section{Rank-Order Preferences with $D$ or $N$ constant in a SET of Games}

Prediction I. Let $G_{N}$ be a set of games, each game of which involves the same number of tosses $N$. Let $\left(D_{1}, N\right),\left(D_{2}, N\right),\left(D_{3}, N\right)$ be members of $G_{N}$ involving denominations $D_{1}<D_{2}<D_{3}$. In an individual's preference ordering within 
$G_{N},\left(D_{2}, N\right)$ cannot be less preferred than both $\left(D_{1}, N\right)$ and $\left(D_{3}, N\right)$. This prediction follows from the assumptions that risk increases as $D$ increases, and that preference is mediated by a single-peaked function over the risk scale.

Prediction II. Let $G_{N}$ and $G_{N^{\prime}}$ be sets of games involving fixed numbers of tosses $N$ and $N^{\prime}$, respectively. Let $\left(D_{i}, N\right)$ be the most preferred (ideal) game in $G_{N}$, and similarly $\left(D_{j}, N^{\prime}\right)$ in $G_{N^{\prime}}$. If $N>N^{\prime}$, then $D_{i} \leqslant D_{j}$. If $N<N^{\prime}$, then $D_{i} \geqslant D_{j}$ (see "Appendix").

Prediction III and IV. Similar predictions hold if one considers a set of games $G_{D}$ involving a fixed denomination $D$, interchanging the roles of $N$ and $D$ in Predictions I and II.

Prediction $V$. Let $\left(D_{i}, N\right)$ be the ideal game in a set $G_{N}$ involving a fixed number of tosses $N$. Let $\left(D, N_{j}\right)$ be the ideal game in a set $G_{D}$ involving a fixed denomination $D$. If $D_{i}>D$, then $N_{j} \geqslant N$. If $D_{i}<D$, then $N_{j} \leqslant N$.

Justification of Predictions II, IV, and V are given in the "Appendix". Figure 1 facilitates an understanding of these predictions. It portrays a space of games where the displayed rows and columns of points are actually sets $G_{N}$ and $G_{D}$, respectively.

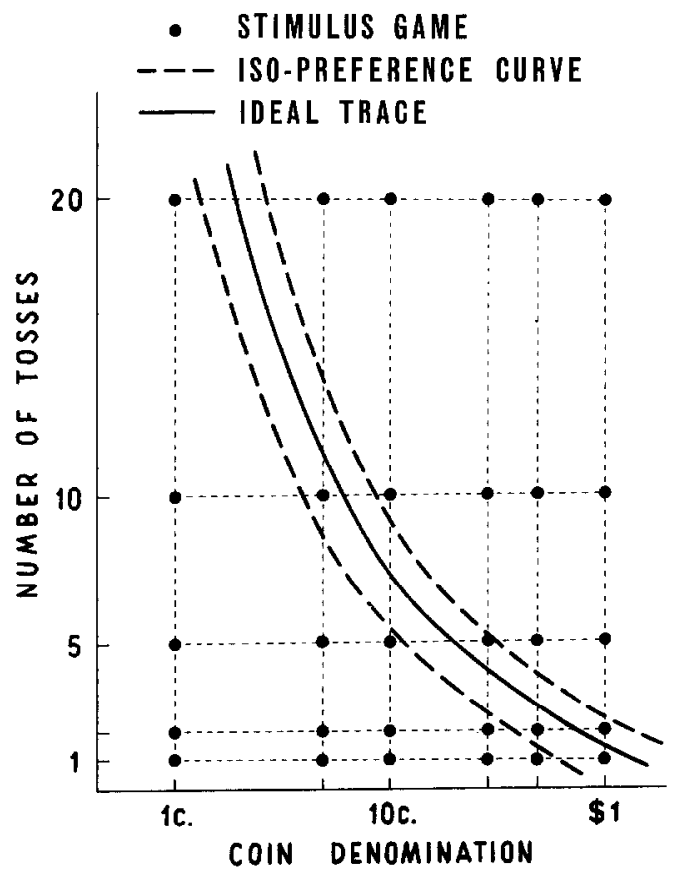

FIG. 1. The space of possible coin-toss games, stimulus games, and subjective iso-preference curves. 
The iso-preference curve over games having risk $R_{0}[(D, N)]=I$, which we call the ideal trace, represents those games having ideal risk. Other games having a constant non-ideal risk level fall on different iso-preference curves, those above the ideal trace reflecting risk larger than the ideal, and those below the trace reflecting risk less than the ideal. Within a set of games, the one falling nearest the ideal trace should be maximally preferred. Predictions II, IV, and V essentially assert that ideal games in different sets must mirror a single monotonically decreasing ideal trace through this space.

\section{Dependency of Portfolio Allottment on Total $N$}

Prediction VI. Suppose an individual must toss for denomination $D_{1}$ and/or $D_{2}$ a total of $N$ times, but that he may choose the numbers $N_{1}$ and $N_{2}$ of times for which $D_{1}$ and $D_{2}$ are respectively tossed, so long as $N_{1}+N_{2}=N$. Further, let $D_{1}<D_{2}$, so that a game with $N-n$ tosses for $D_{1}$ would be less risky than a game with $N-n$ tosses for $D_{2}$. Our Axioms A1 and A3 predict that the proportion of tosses $N_{2} / N$ allotted to the riskier game involving $D_{2}$ must not increase as $N$ increases. This follows because (a) the riskiness of a mixture of two games which differ in riskiness will vary directly with the proportion of tosses in the more risky game of the mixture, if the total number of tosses of both games is fixed, [Al(iii)], and (b) will vary directly with the total number of tosses, if the proportion $N_{2} / N$ is fixed, [Al(i)]. As $N$ increases, the proportion of tosses $N_{1} / N$ in the less risky game $\left(D_{1}\right)$ must increase to maintain an overall risk level closest to $I$. We speak of this as a monotone shift in portfolio balance with total number of tosses.

In fact, our axioms make an even stronger prediction. If $N_{1}=1$, then $N_{2}$ itself must not increase as $N$ increases. (A high risk taker, i.e., one who allots $N_{2}=N$ tosses to the riskier $D_{2}$, might continue with the allottment $N_{2}=N$ as $N$ increases.) This holds because, for any given $N$, the allottment of $N_{1}$ and $N_{2}$ tosses between two games will be that mixture which most closely yields the individual's ideal risk level. To increase $N$ can only increase the total risk of the mixture, no matter how the additional tosses are allotted, hence the additional tosses must all be allotted to the less risky game of the mixture, keeping the mixture's overall risk as close to $I$ as possible.

\section{Prior Commitment}

Prediction VII. If an individual were asked outright to make an absolute estimate about the number of times $(N)$ he would commit himself to toss for a given denomination $(D)$ in a game, this estimate should vary inversely with the proposed denomination.

\section{Equated Pairs of Games}

Prediction VIII. Two games such as "toss for a dime 10 times" and "toss for a dollar 1 time" are called equated games, because they have the same expectation and 
the same extremc outcomcs, i.c., win or losc a dollar. By our axioms concerning $R(\cdot, \cdot)$ and $R_{0}(\cdot)$, to toss more often for a smaller denomination, e.g., toss 10 times for a dime, is less risky than to toss less often for a larger denomination, e.g., toss once for a dollar. Now suppose an individual prefers the less risky of two equated games, e.g., $A$ preferred to $B$. Then by Axiom $A 3$ he must prefer the less risky of two other equated games, e.g., $C$ preferred to $D$, if $C$ is riskier than $A$ and $D$ is riskier than $B$. This follows because the preference of $A$ over $B$ places the optimum risk level in such a manner that $C$ must then be closer to it than is $D$. For example, if an individual prefers tossing twice for a nickel to tossing once for a dime, then he must prefer tossing four times for a quarter to tossing twice for half-a-dollar.

An equivalent dual prediction holds if we begin with the riskier $D$ preferred to $C$. That is, if an individual preferred tossing twice for half-a-dollar to tossing four times for a quarter, then he should prefer tossing once for a dime to tossing twice for a nickel.

\section{METHOD}

Subjects. Thirty males were chosen at random from a pool of paid volunteers at the University of Michigan. They were run in groups of 3-5 in a single session lasting about $45 \mathrm{~min}$. Each received a base salary of $\$ 1.25$.

Stimuli. Five numbers of tosses $(N=1,2,5,10,20)$ and 6 denominations $(D=1 \notin, 5 c$. $10 \notin, 25 \notin, 50 \notin, \$ 1)$ were employed to construct sets of games portrayed as rows and columns of points in Fig. 1. Using these games, the experimenter prepared a stimulus booklet, on the pages of which subjects indicated various preferences within sets of games.

Instructions. The subjects were seated around a table and the experimenter read the instructions. The subjects were familiarized with the stimuli by playing representative games. Play consisted of tossing the specified number of coins $(N)$ all at once. The difference between the number of heads and tails determined what the subject won or lost, respectively. The purpose of tossing the coins all at once was twofold: To emphasize that choosing a number of tosses preceded the play itself (the play was not a sequential process), and to eliminate irrelevant preference or aversion for prolonged participation (the prospect of tossing a penny 20 times might be perceived as rather boring, or as a delightful prospect, irrespective of the risk involved).

After practice and familiarization, the subject was informed that one of the options for which he had shown some preference would be chosen at the end of the session, played for real, and the outcome added to or subtracted from his wages. The purpose of this instruction, aside from the usual invocation of reality, was to convey and emphasize that each decision or choice was to be considered by the subject as an isolated one, independent of all others. We wished to avoid any cumulative effects on preference of building a "portfolio" of games.

At this point, each subject was allowed to withdraw from the experiment with $50 \mathrm{c}$ if he had any reservations about further participation, because it was possible, although unlikely, for him to lose more money than his wages. The remaining subjects (onc withdrew and was replaccd) then received their full wages and the experiment proceeded.

Procedure. Subjects first indicated their preference order within each triad of the games portrayed in the bottom row of points of Fig. $1(N=1)$ and each triad of the third column $(D=10 \notin)$. The triads appeared one per page in the stimulus booklet. These orderings within 
triads were later decomposed (Coombs, 1964, pp. 53-54) and the individual's total preference ordering obtained by weak stochastic transitivity, which was satisfied by everyone. The games in remaining rows and columns (fixed $N$ and $D$, respectively) were printed one row (or column) to a page in one random order administered to all subjects, so that each game occurred on two pages, once as an element of a set having fixed $N$, and once as an element of a set having fixed $D$. The subject was instructed to indicate his preference order on each page by writing the integers $1,2, \ldots$, in front of the games.

Each subject next allotted totals of 5, 10, and 20 tosses between nine selected pairs of denominations used in generating the stimulus games. One pair was printed to a page, and the subject went through all nine, allotting first 5 tosses, then 10 , and then 20 between the member denominations of a pair. Each time, the nine pairs were in a different order. The pairs of denominations were selected to vary the riskiness of the resulting games. Thus, tossing for a penny and for a nickel $(1 \notin, 5 \notin)$ was the least risky pairing. The other eight pairings were $(1 \notin, 10 \notin)$, $(5 \notin, 10 \notin),(5 \notin, 25 \phi),(10 \notin, 25 \phi),(10 \notin, 50 \phi),(25 \phi, 50 \phi),(25 \notin, \$ 1)$, and $(50 \notin, \$ 1)$. Assuming that risk increases with $D$, this listing is in the order of increasing risk.

The subject was then asked to specify exactly how many times he would like to commit himself to toss at each denomination, given that play would only involve the particular denomination for the committed number of tosses.

Finally, subject made pair comparison choices between members of 10 equated pairs of games, such as five tosses of a penny vs. one toss of a nickel, i.e., $(1 \phi, 5)$ vs. $(5 c, 1)$. The other nine pairs were $(1 \notin, 10)$ vs. $(10 \notin, 1),(5 \notin, 2)$ vs. $(10 \notin, 1),(5 \notin, 5)$ vs. $(25 \phi, 1),(5 \phi, 10)$ vs. $(50 \notin, 1)$, $(25 \notin, 2)$ vs. $(50 \notin, 1),(5 \notin, 20)$ vs. $(\$ 1,1),(10 \notin, 10)$ vs. $(\$ 1,1)$, and $(50 \notin, 2)$ vs. $(\$ 1,1)$.

At the experiment's end, each subject played one game which he had chosen during the session and the outcome was added to or subtracted from his salary. This game came from a preselected set, known only to the experimenter, which insurcd that no subject could lose more money than his salary.

\section{RESULTS}

The sets of stimulus games are presented as a matrix in Table 1, with two numbers in each cell. The number on the right indicates the number of subjects who chose the corresponding game as their ideal game for that fixed $N$. The number on the left indicates the number of subjects who chose the corresponding game as their ideal game for that fixed $D$. The total for each row or column is the number of subjects who evidenced single-peaked preference functions over risk and thus satisfied Prediction I, or III, respectively, for that set of games.

There were $6 !(=720)$ possible preference orderings for each set of games having a fixed $N$ in Fig. 1. Thirty-two of these would satisfy Prediction I. Hence, the probability of a subject satisfying this prediction by chance is .044 for any one rank order. In a total of 150 rank orders, there were two which violated this prediction, and their deviation was minor. In the case of sets having fixed $D$, there were $5 !(=120)$ possible orderings, of which 16 would satisfy Prediction III. In a total of 180 rank orders, there were again only two which violated the prediction, and their deviation was minor.

To test Prediction II, a rank order correlation (Kendall's $\tau$ ) was computed between $N$ and the $D_{i}$ associated with the ideal game in each set of games $G_{N}$. Prediction II 
TABLE 1

Location of Ideal. Games within Sets of Games Having Constant $N$ and Constant $D$ (nos. of subjects given in each cell on the right and left, respectively)

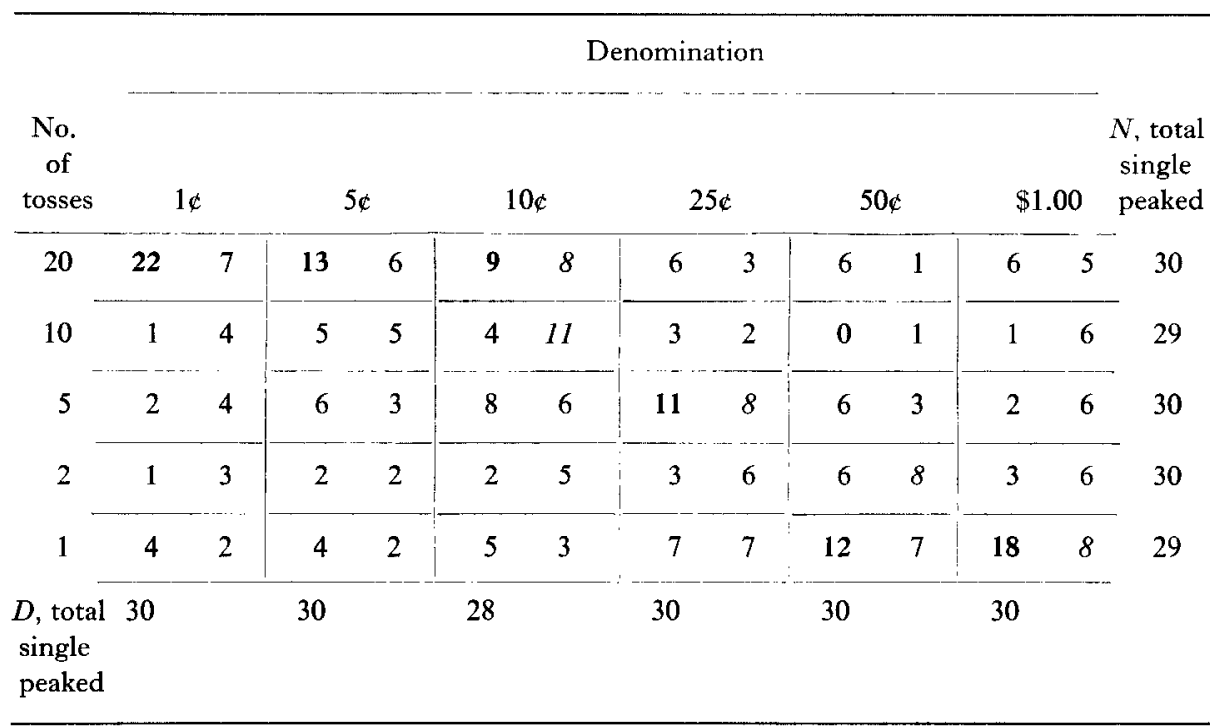

implies a perfect negative correlation $(\tau=-1.0)$. But because of response variability created by error factors, one cannot expect perfect correlation in practice. However, the obtained result should deviate significantly from $\tau=0.0$ (no relation between $N$ and $D$ ). Without extensive replication and further statistical development, it remains extremely difficult to test Prediction II directly. Table 2a presents the results of these computations for 19 subjects. For another 9 subjects, it was impossible to compute a $\tau$ value because of complete ties in the data. Every such instance is compatible, however, with Prediction II. Only 2 subjects failed to lend at least some support to this prediction. Table $2 \mathrm{~b}$ reports similar computations testing Prediction IV. Again there were 9 subjects for whom a $\tau$ value could not be computed because of complete ties in the data.

An important requirement (Prediction V) was that the two kinds of ideal games (ones from sets having variable $D$ with fixed $N$, and ones from sets having variable $N$ with fixed $D$ ) should mirror a single ideal trace for any $S$, as portrayed in Fig. 1. This requirement was tested by considering the relative locations of these ideal games in the space of Fig. 1.

Two similar tests were possible. In the first of these, the subject's ideal games within sets having fixed $N$ were treated as given $a$ priori. With these ideal games specified, the subject's ideal games within sets having fixed $D$ are restricted to only certain 
TABLE 2a

Rank Order Correlation of $D$ with $N$ for Ideal Games from Sets Having Fixed $N$

\begin{tabular}{rcccccc}
$\tau$ & -1.0 & -.87 & -.74 & -.60 & -.47 & \\
No. of subjects & 5 & 5 & 5 & 2 & 2 & 19 \\
Significance level & .001 & .008 & .03 & .07 & .14 & \\
\hline
\end{tabular}

TABLE $2 b$

Rank Order Correlation of $N$ with $D$ for Ideal Games from Sets Having Fixfo $D$

\begin{tabular}{rccccc}
$\tau$ & -1.0 & -.8 & -.6 & -.4 & \\
No. of subjects & 6 & 9 & 2 & 2 & 19 \\
Significance level & .008 & .04 & .12 & .24 & \\
\hline
\end{tabular}

possibilities by Prediction V. (For example, if a subject most preferred (25e, 5) when choosing from games involving five tosses, then when choosing from games involving a $10 \&$ denomination, his ideal game $(10 c, N)$ must have $N \geqslant 5$.) With $D$ fixed, the games of a set were ordered by their $N$ values.

Discrepancies from Prediction V were measured by the number of positions in this order an ideal game deviated from a game compatible with the prediction. (In the above example, if a subject's ideal game was $(10 \mathbb{e}, 2)$ when choosing from games involving a $10 \notin$ denomination, there would be a resulting discrepancy of at least one matrix cell position between the ideal games involving fixed $N$ and $D$.) The magnitude of these discrepancies was used to test the hypothesis that ideal games from the sets having fixed $D$ were unrelated to ideal games from sets having fixed $N$, i.e., that any game from a set having fixed $D$ was equally likely to be ideal within the set. If ideal games were unrelated in this fashion, then the occurrence of at least some discrepancy in the ideal game of a set having fixed $D$ would have approximate probability of 0.5 . There were 11 of 30 subjects for whom the "independence" hypothesis could not be rejected at the .05 level. Across subjects, it could be rejected at an infinitesimal level.

The second analogous test treated the subject's ideal games from sets having fixed $D$ as given $a$ priori, and discrepancies were measured in the subject's ideal games from sets having fixed $N$. In this test, there were five subjects for whom "independence" of the two different ideal sets of games could not be rejected. Of course, as previously remarked, our predictions about the relations between sets of ideal games are much stronger than those tested by the rejection of an "independence" hypotheses. Moreover, a satisfaction of Predictions II and IV implies at least some relation between sets of ideal games. However, to test Prediction V with more appropriate statistical methodology would ultimately require unavailable replications and statistical procedures. 
In more descriptive terms, there were actually 15 subjects whose ideal games were perfectly compatible with Prediction $V$, and hence 15 others for whom compatibility was not complete. Seven of these incompatible subjects failed Predictions II and/or IV to some extent $(\tau \neq 1.0$; at most, five of these failures could possibly be attributed to discrepancies in the results obtained from data collected by the method of triads and by the method of rank order). Thus, there were at least eight subjects having $\tau$ values of -1.0 in Tables $2 \mathrm{a}$ and $2 \mathrm{~b}$, who yet violated the requirement that their ideal games reflect a single monotone ideal trace through the space of Fig. 1.

To test the overall group results, the mode of the ideal games is indicated in each row (italics) and column (boldface) of the matrix in Table 1. It is apparent there that no violation of the requirement of a single common ideal trace occurs. When median ideal are used instead, one discrepancy results, involving a cellular distance of 1 . Hence, in a statistical sense, the overall results, and the results for most individual subjects substantially supported Predictions I-V. However, there were individual instances of violations which cannot be overlooked.

Table 3 reports, for each pair of denominations, the number of subjects whose shift in portfolio balance (Prediction VI) was monotone decreasing with $N=5,10$, and 20 total tosses. Both the proportion $N_{2} / N$ and absolute number $N_{2}$ of tosses allotted to the riskier denomination are considered.

The test of the weaker prediction, that the proportion of tones allotted to the riskier denomination should not increase, was made to judge just how badly the stronger prediction was violated.

TABLE 3

Dependency of Portfolio Allotment on Total Number of Tosses

Pairs of denominations

$(1,5) \quad(1,10) \quad(5,10) \quad(5,25) \quad(10,25)(25,50)(10,50)(25, \$ 1)(50, \$ 1)$

\begin{tabular}{|c|c|c|c|c|c|c|c|c|c|}
\hline \multicolumn{10}{|l|}{ Proportion } \\
\hline Mon. shift as pred. & 26 & 23 & 21 & 21 & 21 & 21 & 20 & 18 & 20 \\
\hline Viol. of pred. & 4 & 7 & 9 & 9 & 9 & 9 & 10 & 12 & 10 \\
\hline $\begin{array}{c}\text { Av no. of com- } \\
\text { patible pairs }\end{array}$ & 2.77 & 2.57 & 2.53 & 2.53 & 2.43 & 2.47 & 2.23 & 2.17 & 2.47 \\
\hline Significance level & .001 & .001 & .001 & .001 & .002 & .002 & .02 & .02 & .002 \\
\hline \multicolumn{10}{|l|}{ Absolute no. } \\
\hline Mon. shift as pred. 2 & 23 & 22 & 16 & 13 & 14 & 15 & 14 & 16 & 14 \\
\hline Viol. of pred. & 7 & 8 & 14 & 17 & 16 & 15 & 16 & 14 & 16 \\
\hline $\begin{array}{l}\text { Av no. of com- } \\
\text { patible pairs }\end{array}$ & 2.60 & 2.33 & 1.90 & 1.93 & 1.80 & 1.83 & 1.80 & 1.77 & 1.80 \\
\hline Significance level & .001 & .01 & & & & & & & \\
\hline
\end{tabular}


Violations of Prediction VI were also measured in degree for each subject by counting the number of implicit pairs in the rank order of proportions $N_{2} / N$ with total $N$ which were compatible with the predicted rank order. Perfectly compatible data would yield 3 compatible pairs, while completely random data would yield 1.5 . The average numbers of compatible pairs are reported in Table 3 for each pair of denominations, and their significance level is indicated where it is of interest, using a normal approximation to the binomial distribution.

A typical example of the violations is the instance in which a subject allotted 5,10 , and 20 tosses between two denominations as follows (risky/less risky): 1/4, 1/9, and $3 / 17$. The 20 tosses should have been distributed in the ratio of not more than $2 / 18$ to maintain a nonincreasing proportion, and 1/19 to maintain a nonincreasing absolute number of tosses for the riskier denomination. Our analysis is conservative in that the violations were often minimal, e.g., a violation of $3 / 17$ instead of a compatible 2/18.

Prediction VII was tested by calculating a rank order correlation for each subject between the number of times to which he had committed himself to toss and the proposed denomination $D$. The distribution of $\tau$ is reported in Table 4 . The four subjects not reported in the table had a constant preferred number of tosses for all denominations. Such subjects do not, of course, violate the prediction.

TABLE 4

Rank-Order Correlation between Committed Number of Tosses and Proposed Denomination

\begin{tabular}{|c|c|c|c|c|c|c|c|}
\hline$\tau$ & -1.00 & -.87 & .74 & -.33 & -.06 & +.33 & \\
\hline No. of subjects & 14 & 4 & 3 & 2 & 2 & 1 & 26 \\
\hline Significance level & .0014 & .0083 & .028 & .235 & .50 & .235 & \\
\hline
\end{tabular}

The maximum number of possible incompatibilities (Prediction VIII) among preferences on equated pairs of games was 25 for each subject. The expected number of incompatibilities would be nine if choices were unrelated across pairs, and choice probabilities between the members of each pair were 0.5 . Just six of the subjects revealed any incompatibilities at all, and the numbers of incompatibilities for these subjects were $1,1,2,2,4$, and 8 , respectively. Only the latter two subjects were within the range in which random response assumptions could not be rejected at the .05 level, and the data lend rather strong support to the prediction.

\section{DISCUSSION}

The overall confirmation of predictions was substantial. This suggests that subjects' choices are mediated by a single-peaked preference function over a unidimensional risk scale, and that there exists a function $R_{0}[(D, N)]$ which is monotone increasing 
with both $D$ and $N$, mapping games onto the risk scale. Preference data for equated pairs of games support the assumption that $D$ is a more powerful risk-determiner than $N$ in the sense of Axiom A1(ii) and Axiom Al(iii), at least over the ranges tested in this experiment. However, it is important to examine individual failures to confirm our predictions, particularly those which violated the existence of a single ideal trace (Prediction V) and appeared to be systematic, rather than caused by random error. These failures may be described as follows: Ideal games within sets in which $D$ was constant reflected an ideal trace, the negative slope of which was too near zero to be compatible with ideal games from sets in which $N$ was constant. Equivalently, ideal games within sets in which $N$ was constant reflected an ideal trace, the negative slope of which was too far from zero to be compatible with ideal games from sets in which $D$ was constant. For example, several subjects had ideal games which involved ranges from 1 to 20 tosses as the fixed $D$ changed from $\$ 1$ to $1 \phi$, whereas the ideal games from sets involving a fixed $N$ of from 1 to 20 tosses ranged only from $25 c$ to $10 \mathrm{c}$. Such subjects maximally satisfied Predictions I-IV, but violated Prediction V. The most extreme form of such a violation is typified by a subject whose ideal games from sets having fixed $N$ were all constant at $D=25 c$, and whose ideal games from sets having fixed $D$ were all constant at $N=5$. Such a subject satisfies Predictions I-IV, but maximally violates Prediction V.

In effect, two different ideal traces were revealed in these failures to satisfy Prediction $\mathrm{V}$, when either $D$ or $N$ was held constant in a set of games. The intersecting locus of ideal games from these different sets suggests that, rather than a shift in the ideal risk level causing the failures, subjects may have evaluated a game's risk by $R_{0}[\cdot]$ when $N$ was constant within a set of games, and by $R_{0}{ }^{\prime}[\cdot]$ when $D$ was constant. An examination of the functions measuring the variance, maximum possible loss, and expected loss of these games with $N$ and $D$ suggests that $R_{0}[\cdot]$ could have involved expected loss, while $R_{0}^{\prime}[\cdot]$ could have involved either variance or maximum possible loss. It is not clear why subjects should choose to adopt two different decision rules for evaluating risk, depending on whether $D$ or $N$ was fixed. Neither is it particularly satisfying to assume that they did so. While it is possible to construct a quantitative theory of preference under which it would be possible for an individual to prefer always to toss for $25 \mathrm{c}$ regardless of the number of tosses involved, or always to toss exactly five times, regardless of the denomination involved, such behavior is not natural for any of the current theories in which the outcomes and the probabilities of outcomes are relevant variables. Such behavior indicates idiosyncratic preference, superstition, or a decision rule chosen by subject to minimize his effort in the experiment.

Subjects also substantially confirmed an overall dependency of pairwise portfolio allottment of number of tosses on the total number of tosses required. However, for all denominational pairs except the two least risky ones, they violated the stronger prediction of no increase in the absolute number of tosses increased. Most of the violations reflect a tendency to maintain more balance than called for in the allottment 
between the two denominations, perhaps reflecting an effect of the instructions to "allot $N$ tosses." Such an effect would be more salient for riskier denominational pairs, where there is more imbalance in the allotment to begin with, and the data provide evidence of this salience.

A general interpretation, compatible with the results here, would be that risk is monotone with some measure of dispersion in possible gambling outcomes. This interpretation of course assumes that expected value is being held constant at all times. For the simple games used in the present study, such dispersion directly varied with both denomination and number of tosses, while expected value was constant at zero in all mixtures of the games. For more complex stimuli, particularly for games having a nonzero expectation, we might not expect risk to be monotone with $D, N$, or more generally with dispersion.

\section{APPENDIX}

Applying Axiom A3, let $P(r)$ be a single peaked preference function over the risk scale $r$, with maximum at $r=I$. Let $R_{0}[(D, N)]$ be the risk of a coin-toss game involving $N$ tosses for denomination $D$.

Proof. (Prediction II). Let $\left(D_{i}, N\right)$ be the ideal game in $G_{N}$, and $\left(D_{j}, N^{\prime}\right)$ the ideal game in $G_{N^{\prime}}$.

Case 1a. Let $R_{0}\left[\left(D_{i}, N\right)\right] \geqslant I$, and $N>N^{\prime}$. We suppose $D_{j}<D_{i}$ and show that

$$
P\left\{R_{0}\left[\left(D_{i}, N^{\prime}\right)\right]\right\}>P\left\{R_{0}\left[\left(D_{j}, N^{\prime}\right)\right]\right\}
$$

which means that $\left(D_{j}, N^{\prime}\right)$ cannot be the ideal game in $G_{N^{\prime}}$. Hence it must be true that $D_{i} \geqslant D_{i}$.

Suppose $D_{j}<D_{i}$. Then we have

(i) $R_{0}\left[\left(D_{j}, N^{\prime}\right)\right]<R_{0}\left[\left(D_{i}, N^{\prime}\right)\right]<R_{0}\left[\left(D_{i}, N\right)\right]$ by the first part of Axioms $\mathrm{A}_{1}$ and $\mathrm{A}_{2}$.

(ii) $R_{0}\left[\left(D_{j}, N\right)\right]<R_{0}\left[\left(D_{i}, N\right)\right]$ and $R_{0}\left[\left(D_{i}, N\right)\right] \geqslant I$ imply that $R_{0}\left[\left(D_{j}, N\right)\right]<I$. Otherwise $P\left\{R_{0}\left[\left(D_{j}, N\right)\right]\right\}>P\left\{R_{0}\left[\left(D_{i}, N\right)\right]\right\}$ since $P(r)$ is single-peaked by Axiom A3, and thus $\left(D_{i}, N\right)$ would not be most preferred in $G_{N}$.

(iii) $R_{0}\left[\left(D_{j}, N^{\prime}\right)\right]<R_{0}\left[\left(D_{j}, N\right)\right]$ and $R_{0}\left[\left(D_{j}, N\right)\right]<I$ imply that

$$
P\left\{R_{0}\left[\left(D_{j}, N^{\prime}\right)\right]\right\}<P\left\{R_{0}\left[\left(D_{j}, N\right)\right]\right\}
$$

since $P(r)$ is single-peaked.

(iv) $P\left\{R_{0}\left[\left(D_{j}, N^{\prime}\right)\right]\right\}<P\left\{R_{0}\left[\left(D_{i}, N\right)\right]\right\}$ since $P\left\{R_{0}\left[\left(D_{j}, N\right)\right]\right\}<P\left\{R_{0}\left[\left(D_{i}, N\right)\right]\right\}$. 
(v) The results of (i) and (iv) imply $P\left\{R_{0}\left[\left(D_{j}, N^{\prime}\right)\right]\right\}<P\left\{R_{0}\left[\left(D_{i}, N^{\prime}\right)\right]\right\}$ since $P(r)$ is single-peaked.

This is the desired contradiction.

Case 1b. Let $R_{0}\left[\left(D_{i}, N\right)\right]<I$ and $N>N^{\prime}$.

Case $2 \mathrm{a}$ and $2 \mathrm{~b}$. Let $N<N^{\prime}$.

The proofs are analogous for these cases.

Proof (Prediction IV). Interchange the roles of $D$ and $N$ in the proof of Prediction II.

Proof (Prediction V). Let $\left(D_{i}, N\right)$ be most preferred in $G_{N}$, and $\left(D, N_{j}\right)$ most preferred in $G_{D}$.

Case 1a. Let $R_{0}\left[\left(D_{i}, N\right)\right] \geqslant I$ and $D_{i}>D$. We suppose that $N_{j}<N$ and show that

$$
P\left\{R_{0}\left[\left(D, N_{j}\right)\right]\right\}<P\left\{R_{0}[(D, N)]\right\}
$$

which means that $(D, N)$ would be more preferred than $\left(D, N_{j}\right)$ in $G_{D}$. This is a contradiction.

Suppose $N_{j}<N$. By A1 and A2 we have

$$
R_{0}\left[\left(D, N_{j}\right)\right]<R_{0}[(D, N)]<R_{0}\left[\left(D_{i}, N\right)\right] .
$$

As argued above, $I \leqslant R_{0}\left[\left(D_{i}, N\right)\right]$ implies that

$$
R_{0}\left[\left(D, N_{j}\right)\right]<R_{0}[(D, N)]<I .
$$

Since $P(r)$ is single peaked, we thus have $P\left\{R_{0}\left[\left(D, N_{j}\right)\right]\right\}<P\left\{R_{0}[(D, N)]\right\}$.

Case 1b. Let $R_{0}\left[\left(D_{i}, N\right)\right]<I$ and $D_{i}>D$.

Case $2 \mathrm{a}$ and $2 \mathrm{~b}$. Let $D_{i}<D$.

The proofs are analogous.

\section{REFERENCES}

Coomвs, C. H. A theory of data. New York: Wiley, 1964.

Coombs, C. H., and PruitT, D. G. Components of risk in decision making: Probability and variance preferences. Journal of Experimental Psychology, 1960, 60, 265-277.

EDWARds, W. The theory of decision making. Psychological Bulletin, 1954, 51, 380-417.

EDWARDs, W. The prediction of decisions among bets. Journal of Experimental Psychology, 1955, 51, 201-214. 
Luce, R. D., AND Raiffa, H. Games and decisions. New York: Wiley, 1957.

Royden, H. L., SuPpes, P., AND Walsh, K. A model for the experimental measurement of the utility of gambling. Behavioral Science, 1959, 4, 11-18.

Wilcox, G. Strategy analysis. Paper presented at the Psychometric Society Symposium on Risky Decision Theory. March 29, 1967, Madison, Wisconsin.

ReCEIVED: May 31, 1968 